\title{
ON A QUESTION OF M. HOCHSTER
}

\author{
R. C. COWSIK
}

ABSTRACT. It is shown that not all finite integral extensions of a Noetherian integrally closed domain $A$ can be obtained by a process of adjoining integral elements whose monic irreducible equations over the quotient field have all the coefficients in the base ring and passing to $A$-subalgebras of the algebras so obtained.

Let $A$ be a domain and $A^{*}$ its quotient field. Let $K$ be an algebraic closure of $A^{*}$. Let $\mathscr{C}(A)$ be the least class of extensions of $A$ in $K$, closed under the two operations:

(a) passing to an $A$-subalgebra,

(b) adjoining an element whose monic irreducible equation over the quotient field of the domain has all the coefficients in the domain.

In $[\mathbf{H}], \mathbf{M}$. Hochster asks whether every finitely generated extension $C$ of $A$ in $K$, such that $C$ is a finitely generated $A$-module and $C \rightarrow A^{*}=A$, must be in $\mathscr{C}(A)$, or even whether, if $A$ is normal, every finitely generated integral extension of $A$ in $K$ must be in $\mathscr{C}(A)$.

We give here an example of a Noetherian unique factorization domain $A$ and a finitely generated integral extension which does not belong to $\mathscr{C}(A)$.

For this, we first prove the following obvious

Lemma. If $C \in \mathscr{C}(A)$, then the $A$-module inclusion $A \hookrightarrow C$ is $A$-split.

Proof. If $C$ is obtained from an extension $B$ of $A$ such that $A \hookrightarrow B$ is $A$-split, then, by applying either (a) or (b), $A \hookrightarrow C$ is split. For, if $C$ were obtained from $B$ by (a), the restriction of the splitting $B \rightarrow A$ to $C$ gives the splitting $C \rightarrow A$. On the other hand, if $C$ is obtained from $B$ by (b), $C$ is a free $B$ algebra and the inclusion $B \rightarrow C$ is $B$-split. By composing we get a splitting $C \rightarrow A$. Now, by induction on the number of times the operations (a) and (b) are used, the lemma follows.

Let $k$ be a field of characteristic 2 . Let $R=k\left[X_{1} \cdots X_{4}\right]$ be the polynomial ring in 4 variables over $k$. Then $S L(4, k)$ acts on $R$ naturally. Let $G$

Received by the editors February 14, 1972.

AMS (MOS) subject classifications (1970). Primary 13B20.

(c) American Mathematical Society 1973 
be the subgroup of $S L(n, k)$ generated by

$$
B=\left(\begin{array}{llll}
1 & 0 & 0 & 0 \\
1 & 1 & 0 & 0 \\
0 & 1 & 1 & 0 \\
0 & 0 & 1 & 1
\end{array}\right)
$$

Then $G$ is a cyclic group of 4 elements.

Let $A$ be the invariants in $R$ under $G$. By the proposition in [B, p. 655], $A$ is a unique factorization domain which is not Cohen-Macaulay.

Proposition. With the notation as above, the domain $R$ is a finite integral extension of $A$ and $R$ is not in $\mathscr{C}(A)$.

Proof. That $R$ is a finite integral extension of $A$ is clear. We prove the inclusion $A \hookrightarrow R$ is not $A$-split. Since $R$ is finite integral over $A$, $\operatorname{depth}_{R}(R)=\operatorname{depth}_{A}(R)$. But if $A$ were an $A$-direct summand of $R$, then $\operatorname{depth}_{A} R=\operatorname{depth}_{A} A$. But, since $A$ is not Cohen-Macaulay, $\operatorname{depth}_{A} A \supsetneqq$ $\operatorname{dim} A=4$. Also depth $R=4$, a contradiction to the assumption $A \hookrightarrow R$ is $A$-split.

\section{REFERENCES}

[H] M. Hochster, Symbolic powers in Noetherian domains, Illinois J. Math. 15 (1971), 9-27. MR 42 \#5966.

[B] Marie-José Bertin, Anneau des invariants du groupe alterné, en caractéristique 2, Bull. Sci. Math. (2) 94 (1970), 65-72. MR 42 \#1819.

School of Mathematics, Tata Institute of Fundamental Research, Bombay 5, INDIA 